\title{
From Appendage to Crosslinker - Unusual Swelling Behavior in Spiropyran-Modified Hydrogels
}

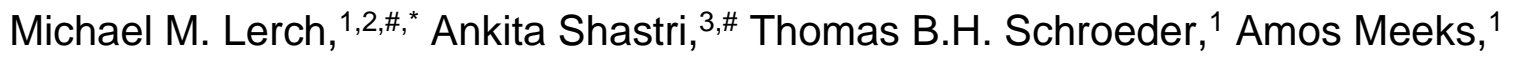 \\ Shucong Li ${ }^{3}$ Anna V. Shneidman, ${ }^{1}$ Michael Aizenberg,${ }^{1}$ Joanna Aizenberg ${ }^{1,3,}{ }^{*}$ \\ 1 John A. Paulson School of Engineering and Applied Sciences, Harvard University, Cambridge, MA \\ 02138, USA; \\ ${ }^{2}$ Stratingh Institute for Chemistry, University of Groningen, Nijenborgh 7, 9747 AG, Groningen, The \\ Netherlands; \\ ${ }^{3}$ Department of Chemistry and Chemical Biology, Harvard University, Cambridge, MA 02138, USA; \\ \# equal contributions \\ * corresponding authors: *jaiz@seas.harvard.edu \& *mlerch@seas.harvard.edu
}

\section{Abstract}

Stimuli-responsive materials typically contain responsive molecular units that couple an external trigger to a defined macroscale response. Ongoing efforts to boost the versatility and complexity of these responses increasingly focus on multi-stimuli-responsive molecular units and crosslinkers, as these bear the potential to impart self-regulatory behaviors building on cooperative effects and feedback mechanisms. Herein, we study a stimuli-responsive platform consisting of polyacrylamide-based hydrogels with well-known multi-responsive spiropyrans covalently bound as pendant groups or 'non-innocent' crosslinkers. Surprisingly, as compared to their appended counterparts, spiropyran crosslinkers cause up to two-fold larger hydrogel swelling in methylenebisacrylamide-crosslinked poly(acrylamide-co-acrylic acid) hydrogels, despite their increased relative crosslinking density. We seek the origin of this unexpected behavior by employing nanoindentation, swelling studies, and UV-vis spectroscopy to study changes in mechanical properties and in spiropyran isomer distribution as a function of solution $\mathrm{pH}$, comonomer chemistry, and swelling-induced polymer strain. We then estimate the osmotic counterion pressures as a function of spiropyran isomer distribution but find that such pressures alone are insufficient to explain the observed behavior. Charge complexation, cooperative effects between the hydrogel's mechanics and chemistry, and aggregate formation may all be invoked to explain features of the observed 'non-innocence' of spiropyran crosslinkers. Taken together, these insights will aid rational implementation of such responsive crosslinkers in materials design and extend the functionality of existing polymeric materials towards more complex and better tunable behaviors. 


\section{Introduction}

Stimuli-responsive hydrogels are crosslinked polymer networks with high water content that undergo large and precisely tunable volume changes in response to physical and chemical stimuli. ${ }^{1}$ Such hydrogels can be engineered to absorb or expel water upon a change in humidity, temperature, $\mathrm{pH}$, electric field, illumination, or other stimuli. The degree of volume changes at both the local and the global level within the hydrogel can be pre-programmed by judiciously choosing the network architecture, backbone chemistry, dopants, and chemical and entropic driving forces. ${ }^{2-5}$ The ease of fabrication, chemical versatility, and degree of tunability of responses have led to the pervasive adoption of stimuli-responsive hydrogels as size-changing smart materials, ${ }^{6}$ with applications spanning a vast range, including microfluidics, ${ }^{7-9}$ soft robotics, ${ }^{4,5,10}$ 4D printing,,$^{11,12}$ optical components, ${ }^{13}$ soft bioelectronics, ${ }^{14,15}$ drug release platforms,${ }^{16}$ and cell culture scaffolds. ${ }^{17,18}$

Many stimuli-responsive hydrogels have by now been developed, but most employ a network architecture whereby the responsive group is attached to the polymer chain as an appendage. ${ }^{19,20}$ However, opportunities to engineer more complex dynamic behaviors arise when responsive moieties are employed as 'non-innocent' crosslinkers ${ }^{21,22}$ that connect polymer chains, as such architectures couple chemical changes to network dynamics and vice versa. Spiropyrans ${ }^{23-27}$ (Fig. 1a) constitute ideal candidates for such crosslinkers because they can exist as isomers with vastly different physical and chemical properties, and thus isomerization greatly affects the local hydrogel network; simultaneously, as spiropyrans are responsive to multiple classes of stimuli, including mechanical strain, ${ }^{28,29}$ polymer swelling can, in turn, impose shifts in chemical equilibrium that influence the molecules' sensitivity to other inputs and that feed back into the polymer network.

Spiropyrans isomers exhibit starkly differing geometry, polarity, dipole moment, charge, acidity, ${ }^{30,31}$ absorption, and fluorescent properties (Fig. 1a). The stability of these isomers strongly depends on their environment and molecular structure, ${ }^{23,25,32-34}$ so the equilibrium between all interconverting isomers can be influenced by $\mathrm{pH}$, solvent polarity, ${ }^{35}$ temperature, ${ }^{36,37}$ light, ${ }^{38}$ analytes, ${ }^{39}$ and mechanical force. ${ }^{28,29}$ In aqueous environments, the polarity of the solvent stabilizes the hydrophilic, strongly colored, ring-open merocyanine form (MC) or its protonated analogue $\left(\mathbf{M C H}^{+}\right)$, depending on the $\mathrm{pH}$ of the environment; yet substantial amounts of the hydrophobic, colorless spiropyran form (SP) are still present, particularly at higher $\mathrm{pH}^{40,41}$ The ring-open $\mathbf{M C}$ and $\mathbf{M C H}^{+}$forms can undergo ring-closure to $\mathbf{S P}$ with visible light or with an increase in hydrophobicity of the surrounding microenvironment. In turn, UV light, heat, or mechanical force can be used to ring-open the $\mathbf{S P}$ isomer. 


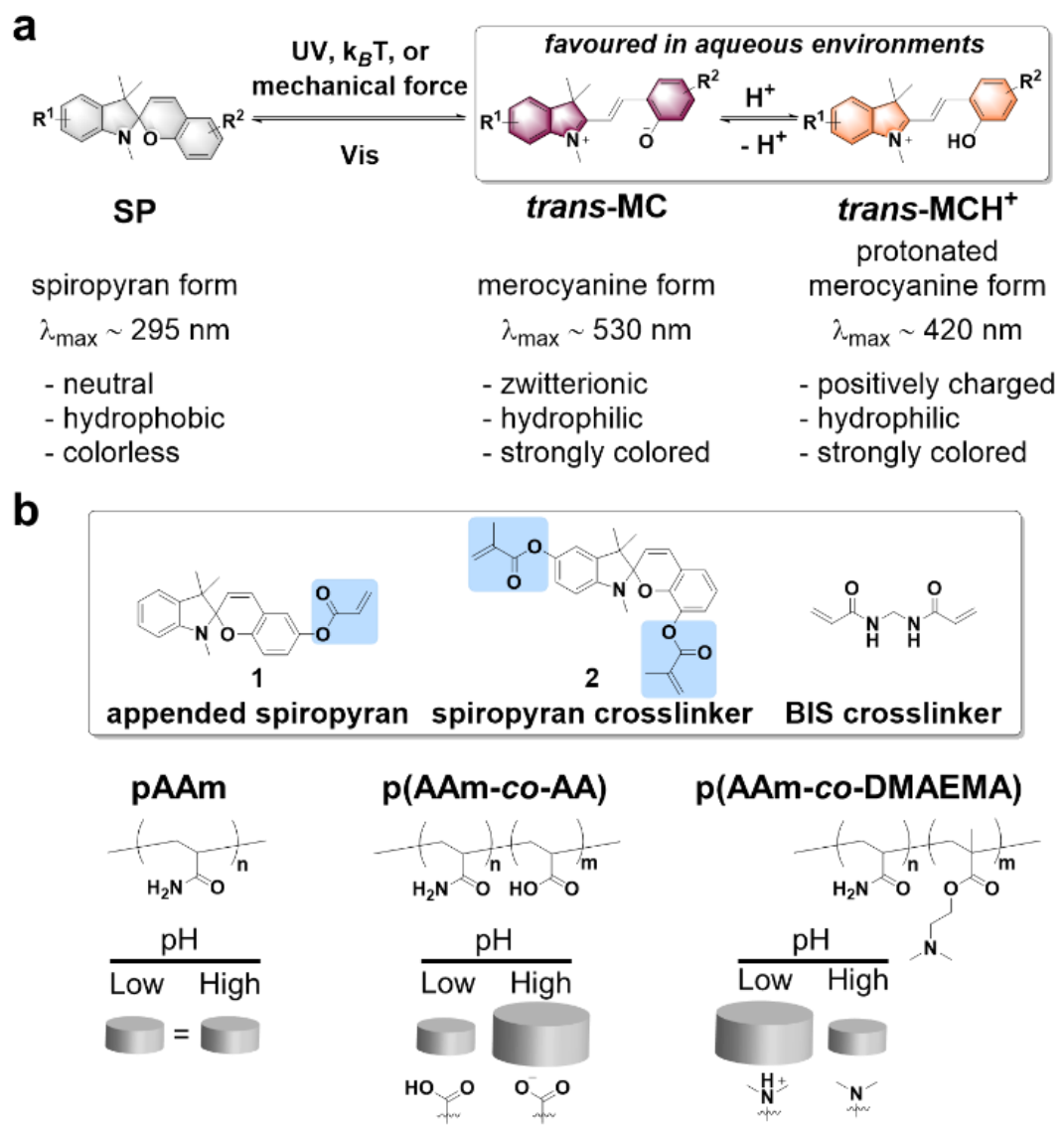

Figure 1. Spiropyran-based hydrogels considered in this work: a) most relevant spiropyran isomers (SP, $\mathbf{M C}$ and $\mathbf{M C H}^{+}$) and their properties. b) Spiropyrans as an appendage (1) or as a crosslinker (2) were studied in three types of hydrogels with different $\mathrm{pH}$-dependent swelling-behavior: unresponsive pAAm (polyacrylamide), acidic $\mathrm{p}(\mathrm{AAm}-\mathrm{co}-\mathrm{AA})$ gels (poly(acrylamide-co-acrylic acid)) that swell with higher $\mathrm{pH}$, and basic $\mathrm{p}(\mathrm{AAm}-\mathrm{co}-\mathrm{DMAEMA})$ gels (poly(acrylamide-co-2-(dimethylamino)ethyl methacrylate)) that swell at lower $\mathrm{pH}$.

For spiropyrans pendant to a hydrogel network, changes in charge, dipole moment, and polarity associated with stimuli-triggered switching between isomers (from $\mathbf{M C H}^{+} / \mathbf{M C}$ to $\mathbf{S P}$ ) affect the swelling equilibrium of the polymer matrix. ${ }^{19}$ Adjustments to the appended spiropyran group's molecular structure and charge are commonly utilized to tailor the swelling behavior of these gels. ${ }^{20}$ Fixed charges within the hydrogel matrix trigger volume changes in hydrogels because they require mobile counterions, which cause the osmotic pressure of the hydrogel interior to rise. For more non-polar elastomers, in which the ring-closed SP form dominates, incorporating spiropyrans as crosslinkers allows one to use them as mechanophores: mechanically stretching these polymers triggers ring-opening of the non-colored SP to the colored MC isomer, allowing the ensuing local coloration of the elastomer to function as a strain reporter. ${ }^{42,43}$ Both types of spiropyran behavior are widely used, but the interplay between these (and other) modalities for the creation of self-regulated soft materials has received little attention and remains difficult to 
account for when designing smart systems. Here, we provide insights into this interplay of multiple stimuli-responsive modalities of spiropyrans in polyacrylamide-based hydrogels and demonstrate the utility of spiropyrans as non-innocent crosslinkers (Fig. 1b). We find that hydrogels equipped with a spiropyran-based crosslinker show unexpected large-magnitude swelling, which we attribute to the interplay of mechano-responsivity and $\mathrm{pH}$-responsivity together with an increase in steric constraint around the spiropyran crosslinker. By invoking possible charge complexation effects and cooperativity between the gel's chemical and mechanical properties we provide possible explanations for the observed trends and highlight that non-innocent crosslinkers provide interesting design implications for hydrogel actuators.

\section{Results and Discussion}

\section{An unusual swelling behavior}

The investigations described herein were triggered by the observation of large increases in hydrogel swelling in spiropyran-crosslinked poly(acrylamide-co-acrylic acid) hydrogels (p(AAmco-AA-co-2), Fig. 2) in the context of our efforts to design optically active, self-regulated hydrogels. ${ }^{44,45}$ To this end, spiropyran variants $\mathbf{1}$ and $\mathbf{2}$ were synthesized (SI Sections $\mathbf{1}$ and 2) and co-polymerized into methylenebisacrylamide-crosslinked polyacrylamide hydrogels (SI Section 3, Tables S3.1-S3.3, verification of attachment by FTIR in SI Section 5.1). The spiropyran-free, control hydrogel, $\mathrm{p}(\mathrm{AAm}-\mathrm{co}-\mathrm{AA})$ polymerized at a 1:1 AAm:AA molar ratio and crosslinked with 1.1 mol\% methylenebisacrylamide (BIS), swells in aqueous media with a swelling ratio $(\mathrm{SR}$ ) increasing from $\sim 2$ to $\sim 11$ as $\mathrm{pH}$ increases from $\mathrm{pH} 5.5$ to 7.5 , due to the accumulation of negative charges on the polymer backbone resulting from the deprotonation of carboxylic acid moieties ( $\mathrm{p} K_{\mathrm{a}} \sim 4.7$, Fig. 2, black line). When $\mathrm{p}(\mathrm{AAm}-\mathrm{co}-\mathrm{AA}$ ) hydrogels are copolymerized with 0.4 mol\% spiropyran 1 (Fig. 2, orange line), a similar trend with slightly higher swelling ratios (from $\sim 3$ to $\sim 14)$ above $\mathrm{pH} 5.5$ is observed. Interestingly, when $\mathrm{p}(\mathrm{AAm}-\mathrm{co}-\mathrm{AA})$ hydrogels are copolymerized with 0.4 mol\% spiropyran 2 as an additional crosslinker to the $1.1 \mathrm{~mol} \%$ BIS (Fig. 2, blue line), the resulting hydrogel slabs display dramatically enhanced swelling across the $\mathrm{pH}$ range (e.g. $\sim 7$ vs. $\sim 3$ at $\mathrm{pH} 5.5$ and $\sim 20$ vs. $\sim 13$ at $\mathrm{pH} 6.8$ for $\mathrm{p}(\mathrm{AAm}-\mathrm{co}-\mathrm{AA}-\mathrm{co}-2)$ and $\mathrm{p}(\mathrm{AAm}-\mathrm{co}-$ AA-co-1), respectively). This stark increase in swelling ratio is particularly remarkable given the relatively low concentration of spiropyran $(0.4 \mathrm{~mol} \%)$ and that less swelling might be expected at the higher crosslinking density resulting from the presence of an additional crosslinker 2. 


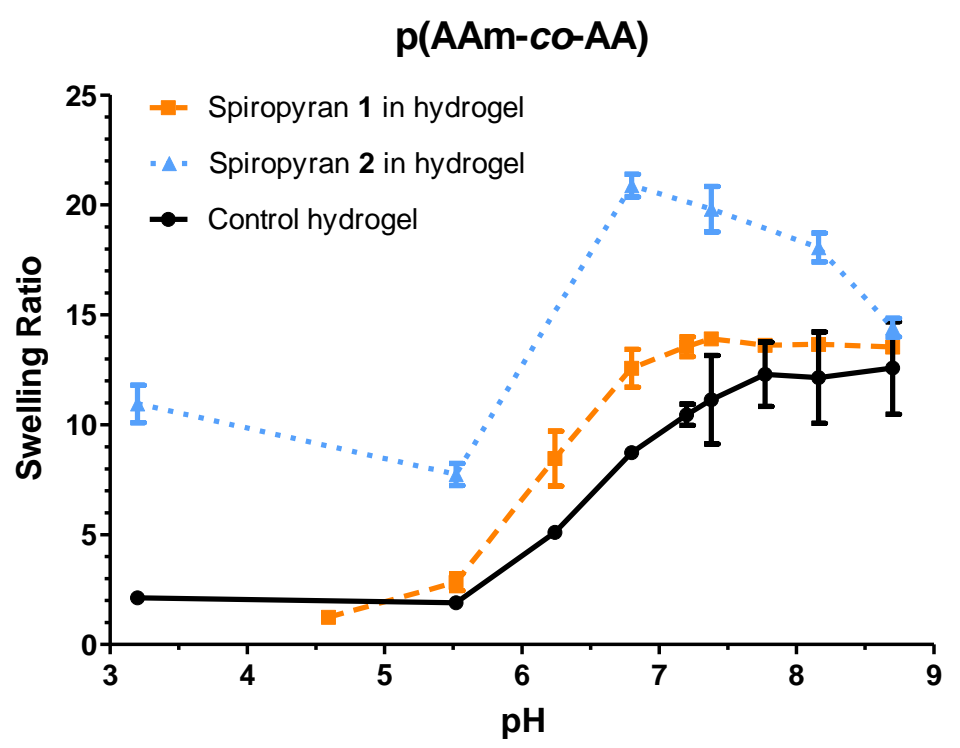

Figure 2. $\mathrm{pH}$-dependent volume change of spiropyran-functionalized $\mathrm{p}(\mathrm{AAm}-\mathrm{co}-\mathrm{AA})$ in $50 \mathrm{mM}$ phosphate buffer, in which spiropyran 1 acts as an appendage and spiropyran 2 as a crosslinker. The swelling ratio (SR) at each $\mathrm{pH}$ was calculated as $\mathrm{SR}=$ (mass swollen - mass dry)/mass dry.

Hydrogel swelling results from a balance of the elastic pressure of the gel network, which favors collapse, with the solvent mixing and ionic osmotic pressure, which drive gel swelling. Changing the degree of crosslinking within a hydrogel will affect the elastic properties of the gel network and thus the extent of swelling at equilibrium. To confirm the observed effect, we measured swelling and mechanical properties in control pAAm hydrogels (SI Section 4). Non-functionalized pAAm hydrogels are expected to undergo no $\mathrm{pH}$-dependent changes in their charged state; they do not significantly change volume across the examined $\mathrm{pH}$ range (Fig. S5.5). Incorporation of spiropyran 2 produces slightly higher swelling ratios across the $\mathrm{pH}$ range. We then performed nanoindentation measurements (Fig. S4.1) to estimate the elastic moduli in order to check whether the additional crosslinks provided by 2 are indeed reflected in the mechanics of the gel (Fig. 3, \& Figs. S4.2 \& S4.3). Comparing pAAm control hydrogels with either $1.1 \mathrm{~mol} \%$ or $2.2 \mathrm{~mol} \%$ BIS crosslinker, but no spiropyran, shows, as expected, that swelling decreases by about $21 \%$ and the elastic modulus increases as crosslinking density increases by about $37 \%$. Incorporating either spiropyran $\mathbf{1}$ or $\mathbf{2}$ significantly decreases the elastic modulus for both $1.1 \mathrm{~mol} \%$ or $2.2 \mathrm{~mol} \%$ BIS-crosslinked hydrogels (Fig. 3; by about $63 \%$ and $43 \%$ for $p$ (AAm-co1) with either $1.1 \mathrm{~mol} \%$ or $2.2 \mathrm{~mol} \% \mathrm{BIS}$, respectively). In addition, p(AAm-co-2) at $1.1 \mathrm{~mol} \% \mathrm{BIS}$ exhibits about a $38 \%$ drop in elastic modulus with respect to the pAAm control, but a $66 \%$ increase with respect to $\mathrm{p}(\mathrm{AAm}-\mathrm{CO}-\mathbf{1})$. This effect persists even after correcting for hydrogel swelling (Fig. S4.4), suggesting an effectively lower crosslinking density, which could possibly be explained by inhomogeneities during free radical polymerization. We further note, that the spiropyran 
crosslinker is about 1.5-fold or 2.2-fold longer than BIS in its SP or MC form, respectively, possibly influencing network dynamics in this way. Nevertheless, comparing $p(A A m-C o-A A-c o-1)$ and $\mathrm{p}(\mathrm{AAm}-\mathrm{co}-\mathrm{AA}-\mathrm{Co}-2)$ at $1.1 \mathrm{~mol} \%$ BIS crosslinking demonstrates that the switch from appendage to crosslinker increases the effective crosslinking density. However, this increase in elastic modulus is not reflected in otherwise expected reduction in swelling, as we observe greater swelling in $\mathrm{p}(\mathrm{AAm}-\mathrm{co}-\mathrm{AA}-\mathrm{co}-2)$ compared to $\mathrm{p}(\mathrm{AAm}-\mathrm{co}-\mathrm{AA}-\mathrm{co}-1)$.

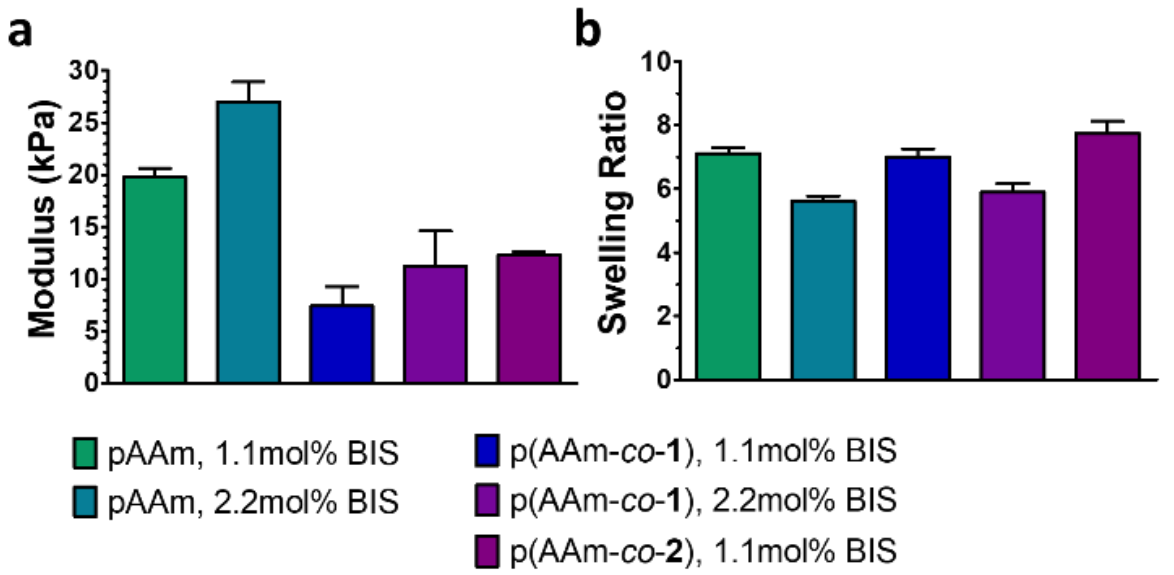

Figure 3. Influence of crosslinking density on elastic moduli and swelling of neutral pAAm hydrogels. a) Elastic moduli of pAAm gels soaked in deionized water measured by nanoindentation. b) Swelling ratio of pAAm gels swollen in PBS $1 \mathrm{X} \mathrm{pH} \mathrm{7.2.}$

Stimuli-responsive swelling/contraction for hydrogels with appended spiropyrans relies on changes in charge and polarity between the positively charged $\mathbf{M C H}^{+}$, zwitterionic (overall neutral) MC, and neutral SP spiropyran isomers. ${ }^{19,20,44}$ We therefore set out to elucidate the isomer distributions for both spiropyran variants at different $\mathrm{pH}$ values in the gel to understand whether the difference in swelling could be explained by differences in isomer distribution (and thus changes in osmotic counterion pressure) alone. To that end, UV-vis spectroscopy was used to identify spiropyran isomer signatures in buffered spiropyran solutions and in hydrogels (SI section 5.3) to understand how solution $\mathrm{pH}$, covalent attachment, and hydrogel volume affect spiropyran isomer equilibria. To put the $\mathrm{p}(\mathrm{AAm}-\mathrm{co}-\mathrm{AA})$ results in context and to experimentally decouple $\mathrm{pH}$ and swelling effects on isomer distributions, we also study neutral polyacrylamide (pAAm) and basic poly(acrylamide-co-2-(dimethylamino)ethyl methacrylate) $p(A A m-c o-D M A E M A)$ ) hydrogels (Fig. 1b), with no and opposite $\mathrm{pH}$-dependent swelling compared to $\mathrm{p}(\mathrm{AAm}-\mathrm{co}-\mathrm{AA})$. We then compare these results to a simple theoretical mode ${ }^{46}$ (SI Section 6) that estimates isomer distribution at different $\mathrm{pH}$ based on Donnan equilibrium theory and compare its estimate of resulting osmotic counterion pressures to experimentally observed swelling (SI Section 5.2). 


\section{Understanding spiropyran 1 isomer distributions}

a

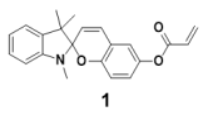

b

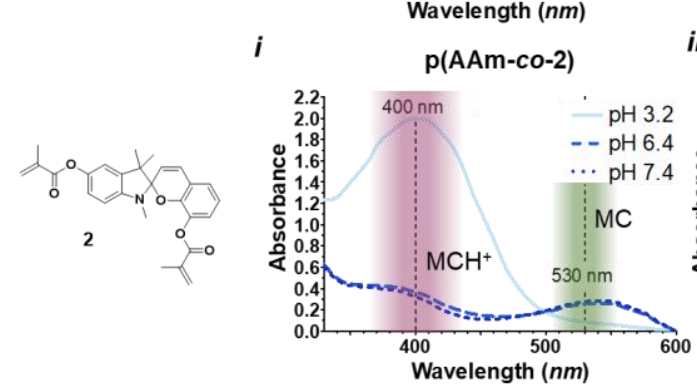

$i$

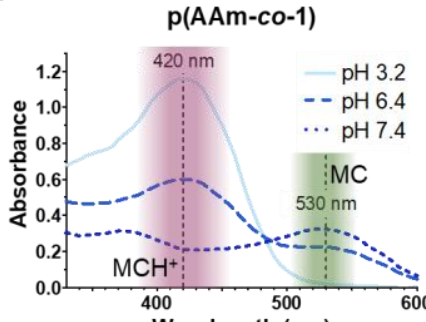

ii

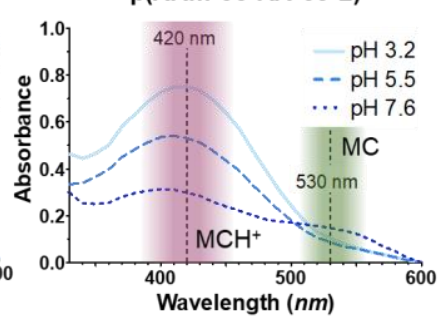

iii

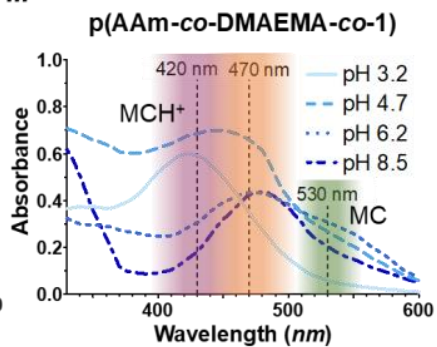

iii

p(AAm-Co-DMAEMA-co-2)

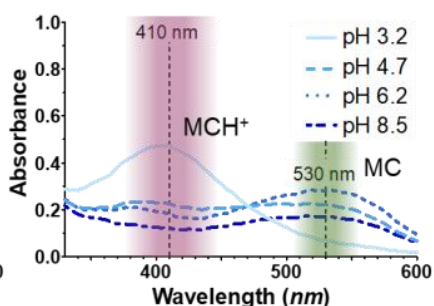

Figure 4. UV-vis spectra of spiropyran-containing hydrogels of different swelling behavior: equilibrium spiropyran isomer distribution for a) spiropyran 1 polymerized into methylenebis(acrylamide)-crosslinked pAAm, $\mathrm{p}(\mathrm{AAm}-\mathrm{co}-\mathrm{AA})$, and $\mathrm{p}(\mathrm{AAm}-\mathrm{co}-\mathrm{DMAEMA})$ hydrogels in the $\mathrm{pH}$ 3-9 range; and b) spiropyran 2 polymerized into methylenebis(acrylamide)-crosslinked pAAm, $p(A A m-c o-A A)$, and $p(A A m-c o-D M A E M A)$ hydrogels in the $\mathrm{pH}$ 3-9 range.

Buffered solutions: We start by determining the $\mathrm{pH}$-dependency of spiropyran isomer distributions in buffered aqueous solutions alone. Compounds $\mathbf{1}$ and $\mathbf{2}$ differ in the $\mathrm{p} K_{\mathrm{a}}$ of the merocyanine phenolic proton $\left(\mathbf{M C H}^{+}\right.$to $\mathbf{M C}$ equilibrium); exhibiting an estimated $\mathrm{p} K_{\mathrm{a}}$ value of slightly above 6 (Fig. S5.17) and 5, respectively. Accordingly, in buffered aqueous solutions (50 mM phosphate buffers), these spiropyrans predominantly exist as the $\mathbf{M C H}^{+}$isomer $\left(\lambda_{\max } \sim 400-\right.$ $430 \mathrm{~nm})$ at low $\mathrm{pH}$ and as $\mathbf{M C}\left(\lambda_{\max } \sim 530 \mathrm{~nm}\right)$ at high $\mathrm{pH}$ (Figs. S5.15 \& S5.16). Moreover, as $\mathrm{pH}$ increases, relatively more SP isomer is likely present ${ }^{47}$ as the equilibrium shifts from $\mathbf{M C H}^{+}$to $\mathbf{M C}$ due to MC-to-SP interconversion. (The absorption band of SP is usually observed below $300 \mathrm{~nm}$ and is difficult to resolve in our measurements as reagents and multi-well measurement plates absorb in this region.)

Spiropyran 1 in a neutral hydrogel: We next co-polymerized compound 1 into BIS-crosslinked, neutral pAAm hydrogels immersed within different buffers in order to confirm that covalent attachment to a polymer backbone did not induce changes in the isomerization equilibrium. Indeed, in p(AAm-Co-1) hydrogels, a similar trend was observed as that of free spiropyran in buffer solution (Fig. 4a, $\boldsymbol{i}$; Fig. S5.19): $\mathbf{M C H}^{+}$is present at low $\mathrm{pH}$ and converts to $\mathbf{M C}$ at higher $\mathrm{pH}$. Swelling is comparable for pAAm and p(AAm-Co-1) gels (Fig. 3b and Fig SI S4.3), indicating that the concentration of charged spiropyran isomers in this neutral gel is not sufficient to induce large swelling effects. 
Effect of ionizable co-monomers on spiropyran 1: The presence of acidic or basic comonomers will influence the isomer distribution through ionization and a buffering effect. ${ }^{33}$ This effect is evident in pre-polymer solutions (compare Figs. S5.18, S5.21 \& S5.24) and crosslinked hydrogels (Fig. 4a,ii \& 4a,iii; Figs. S5.22 \& S5.25), where the presence of acrylic acid increases the stability of $\mathbf{M C H}^{+}$to a higher $\mathrm{pH}(>6)$, whereas the basic DMAEMA moieties destabilize $\mathbf{M C H} \mathbf{C H}^{+}$ even below $\mathrm{pH}$ 5. Because the co-monomer moieties can ionize, their interaction with charged spiropyran isomers may have a stronger effect on hydrogel volume than in the case of neutral pAAm.

Theoretical model: Based on an earlier model, ${ }^{46}$ we implemented a theoretical model based on the Donnan equilibrium to estimate counterion pressures in charged hydrogels as a proxy for swelling (SI Section 6). The estimated relative counterion pressures for non-spiropyran functionalized $\mathrm{p}(\mathrm{AAm}-\mathrm{co}-\mathrm{AA})$ and $\mathrm{p}$ (AAm-co-DMAEMA) hydrogels fit qualitatively well with experimental results. The internal $\mathrm{pH}$ of polyelectrolyte hydrogels is shifted significantly lower (in AA-containing gels) or higher (in DMAEMA-containing gels) compared to the external solution $\mathrm{pH}$ (Figs. S6.3 \& S6.7). Indeed, the swelling at $\mathrm{pH} 5.5$ to 7 for $\mathrm{p}(\mathrm{AAm}-\mathrm{co}-\mathrm{AA})$ hydrogels (control, Fig. 2) is induced by the deprotonation of carboxylic acid groups as the gel's internal pH rises above the $\sim 4.7 \mathrm{pK}$ of acrylic acid. Similarly, the swelling of $\mathrm{p}(\mathrm{AAm}-$ co-DMAEMA) gels below $\mathrm{pH} 7$ (Figs S5.9 \& S5.11) can be correlated with the protonation of the tertiary amine moiety $\left(p K_{a} \sim 8.6^{48}\right)$. Potentially, a small amount of hydrolysis of the DMAEMA/AAm co-monomers to AA may contribute somewhat to the experimentally observed gel contraction for DMAEMA-containing gels at lower $\mathrm{pH}$, which is not accounted for by the model. The change in internal $\mathrm{pH}$ predicted for nonspiropyran-containing gels also fits well with the experimental shifts in $\mathbf{M C H} \mathbf{C H}^{+}$to $\mathbf{M C}$ equilibrium for both $\mathrm{p}(\mathrm{AAm}-\mathrm{co}-\mathrm{AA}-\mathrm{co-1})$ and $\mathrm{p}(\mathrm{AAm}-\mathrm{Co}-\mathrm{DMAEMA-co-1)}$ gels.

Effect of hydrogel volume change on spiropyran 1: In addition to gel internal pH changes, the hydrogel volume change itself could influence spiropyran isomer distribution, as the microenvironment of the spiropyrans conjugated to the polymer will undergo (i) a reduction in the density of surrounding organic molecules, (ii) a reduction in the concentration of buffering groups and neighboring spiropyrans, (iii) an increase in the size of nearby pores, and (iv) an increase in the strain of the polymer network. In order to disentangle these effects, we compared the spiropyran isomer distribution in $\mathrm{p}(\mathrm{AAm}-\mathrm{co}-\mathrm{AA})$ and $\mathrm{p}(\mathrm{AAm}-\mathrm{co}-\mathrm{DMAEMA})$-gels with opposite $\mathrm{pH}$-dependent swelling behavior (Fig. 1b, SI Section 5.2)-keeping in mind the differences in internal pH. $\mathrm{p}(\mathrm{AAm}-\mathrm{co}-\mathrm{AA}-\mathrm{co}-\mathbf{1})$ hydrogels show predominantly $\mathbf{M C H}^{+}$up to $\mathrm{pH}$, similar to the AAm/AA prepolymer solution. In their swollen state at higher $\mathrm{pH}$ ( $\mathrm{pH} 7.8$, Fig. 4a,ii; Fig. S5.22), these hydrogels exhibit an unexpected absorption band at $\lambda_{\max } \sim 470 \mathrm{~nm}$ (for a discussion concerning this peak, vide infra). While a shoulder indicative of the MC isomer at $\lambda_{\max } \sim 530-540 \mathrm{~nm}$ 
is observed in the AAm/AA pre-polymer solution (Fig. S5.21) both at low and high $\mathrm{pH}$, no such band is discernible in the polymerized $\mathrm{p}(\mathrm{AAm}-\mathrm{CO}-\mathrm{AA}-\mathrm{CO}-\mathbf{1})$ absorption spectrum. For $\mathrm{p}\left(\mathrm{AAm}-\mathrm{co}^{-}\right.$ DMAEMA) hydrogels, the basicity of the gel disfavors formation of $\mathbf{M C H}^{+}$, as can be seen in $\mathrm{p}$ (AAm-co-DMAEMA-co-1) hydrogels, in which the $\mathbf{M C H}^{+}$isomer is only observed at $\mathrm{pH}$ 3.2. As the $\mathrm{pH}$ increases to $\mathrm{pH} 8.5$ (dark blue line, Fig. 4a,iii; Fig. S5.25 \& S5.26) and the hydrogel contracts, a shift from $\lambda_{\max } \sim 430 \mathrm{~nm}$ to $\lambda_{\max } \sim 470 \mathrm{~nm}$ is observed. At pH 6.2, an absorption band at $\lambda_{\max } \sim 530 \mathrm{~nm}$ is intermittently detectable, possibly indicating presence of the MC isomer. Overall, both $\mathrm{p}(\mathrm{AAm}-\mathrm{co}-\mathrm{AA}-\mathrm{co-1})$ and $\mathrm{p}$ (AAm-co-DMAEMA-co-1) hydrogels favored $\mathbf{M C H}^{+}$at low $\mathrm{pH}$ consistent with our model. However, the shift towards an absorption band centered at $\lambda_{\max } \sim 470 \mathrm{~nm}$ at higher $\mathrm{pH}$ is unexpected and observed for both hydrogels. As these gels swell at opposite $\mathrm{pH}$ ranges, the formation of the species identified at $\lambda_{\max } \sim 470 \mathrm{~nm}$ seems dependent on $\mathrm{pH}$ and not on swelling, suggesting that hydrogel volume changes have little effect on the isomer distribution of pendant spiropyrans. With regard to the observed swelling behavior, both hydrogels show slightly increased volumes across the $\mathrm{pH}$ range as compared to their non-functionalized counterparts, which is possibly explained by the presence of charged merocyanine isomers to varying degrees at all $\mathrm{pH}$ values.

\section{Understanding spiropyran 2 isomer distributions}

Next, we turned our attention to hydrogels containing spiropyran 2, which, as a crosslinker, is responsive to mechanical pulling forces from the hydrogel network and thus can serve as a mechanophore, in contrast to compound 1. As hydrogels swell, the network is subjected to swellinginduced strain, which can be released by the ring-opening of the cyclic SP isomer to its merocyanine $\left(\mathbf{M C} / \mathbf{M C H}^{+}\right)$counterparts, ${ }^{49}$ possibly changing the isomer distribution. For neutral pAAm hydrogels crosslinked with methylenebisacrylamide and additional spiropyran 2 crosslinker (p(AAm-Co-2), Fig. 4b,i; Fig. S5.20), one would expect few changes in spiropyran isomer distribution compared to $\mathrm{p}(\mathrm{AAm}-\mathrm{co}-\mathbf{1})$. Indeed, the $\mathbf{M C H}^{+}$isomer dominates at low $\mathrm{pH}$ in favor of the MC-isomer at higher $\mathrm{pH}$. Introduction of the second (meth)acrylate group in spiropyran 2 compared to spiropyran 1 changes the electronics of the system, slightly lowering the effective $p K_{a}$ of the phenolic proton (vide supra, Fig. S5.17), which is particularly apparent in the spectra taken at $\mathrm{pH} 6.4$ (Fig. $\mathbf{4 a}, \mathbf{i}$ vs. $\mathbf{4 b}, \mathbf{i}$ ). In addition, the altered electronics cause the $\mathbf{M} \mathbf{C H}^{+}$absorption band to slightly shift hypsochromically from $\lambda_{\max } \sim 420 \mathrm{~nm}$ to $\lambda_{\max }=400 \mathrm{~nm}$ (Figs. S5.15 \& S5.16). Nevertheless, experimentally $\mathrm{p}(\mathrm{AAm}$-co-2) hydrogels swell more (Fig $\mathbf{3 b}$ ) in a manner that cannot be explained by the changes in isomer distribution but hints at an explanation invoking other types of effects, as discussed below. P(AAm-co-AA-co-2) hydrogel samples (Fig. 4b,ii; Fig. S5.23) show similar isomer distributions as $\mathrm{p}\left(\mathrm{AAm}-\mathrm{Co}^{-2}\right)$ (Fig. $\left.\mathbf{4 b}, \boldsymbol{i}\right)$, taking the generally lower internal $\mathrm{pH}$ of 
$\mathrm{p}\left(\mathrm{AAm}-\mathrm{co}-\mathrm{AA}\right.$ ) gels (Fig. S6.3) and thus later onset of $\mathbf{\mathbf { M C H }}{ }^{+}$deprotonation into account. As the gel swells with increasing $\mathrm{pH}$, one would expect that strain-promoted ring-opening of $\mathbf{S P}$ isomers would lead to an increase in charged species in the system, which could further swell the gel (vide infra). However, a relative increase in merocyanine isomers is difficult to verify solely from UV-vis spectra.

In order to estimate whether the observed spiropyran isomer distribution for spiropyrans $\mathbf{1}$ and $\mathbf{2}$ could be used to explain the changes in swelling trends in $p(A A m-c o-A A)$ gels containing spiropyrans shown in Fig. 2, the charges of the different spiropyran isomers were included in our theoretical model (SI Section 6). Estimating counterion pressures in gels containing spiropyran $\mathbf{1}$ (the model does not include any mechanical effects) suggests, counter to our observations (Fig. 2 ), that the presence of spiropyran should reduce swelling throughout the $\mathrm{pH}$ range but that these effects should be negligible given the low concentration of spiropyran (Figs S6.2 \& S6.6). This suggests that the observed swelling trends cannot be simply explained by counterion pressures stemming from charges of spiropyran isomers alone. It is worth noting, that our model assumes spatial homogeneity (i.e. perfect charge balance); however, strongly charged polyelectrolytes cause inhomogeneity, ${ }^{50-53}$ leading to deviations from the Donnan equilibrium that could contribute to discrepancies between theoretical predictions and experimental results. In addition, for highly swollen $(S R>50)$ and charged polymers, counterion pressures normally dominate, but for lower swelling ratios, as in the present system, mixing pressures have to considered as well. ${ }^{54}$ Therefore, other possible explanations must be considered. We have identified three possible contributors: (i) charge complexation, (ii) cooperative chemo-mechanical effects, and (iii) aggregate formation, which together may explain the observed effects.

\section{Identifying factors contributing to the observed swelling behavior}

Charge complexation: Every charged group in a polyelectrolyte solution must be balanced by a counterion of opposite charge. These counterions may be mobile or fixed to a polymer chain. Charge-charge interactions between appended $\mathbf{M C H}^{+}$and the deprotonated carboxylic acid in $\mathrm{p}(\mathrm{AAm}-\mathrm{co}-\mathrm{AA}-\mathrm{Co}-1)$ may reduce the mobile counterion pressure at internal $\mathrm{pHs}$ between the $\mathrm{p} K_{\mathrm{a}} \mathrm{S}$ of $\mathbf{M C}$ and $\mathrm{AA}$, keeping swelling moderate in this intermediate $\mathrm{pH}$ range. For the more sterically hindered spiropyran crosslinker 2 in p(AAm-co-AA-co-2), however, such intermittent charge-charge stabilization may not be possible, thus greatly increasing the mobile counterion pressure (and, in turn, swelling) by comparison. Charge complexation (or lack thereof) can thus be invoked to explain why $p(A A m-c o-A A-c o-1)$ swells to similar extent to the $p(A A m-c o-A A)$ control, but $p$ (AAm-co-AAco-2) swells much more at low to intermediate $\mathrm{pH}$ (Fig. 2). This is corroborated by swelling trends in $\mathrm{p}$ (AAm-Co-DMAEMA) hydrogels (Figs. S5.9 \& S5.11), in which the positive charge of $\mathbf{M C H}^{+}$ cannot be counteracted by negative charge from the backbone. An argument against this 
explanation is that $\mathrm{p}(\mathrm{AAm}-\mathrm{co}-\mathrm{AA}-\mathrm{co}-\mathbf{1})$ hydrogels at $2.2 \mathrm{~mol} \% \mathrm{BIS}$ swell more in this $\mathrm{pH}$ range (Fig. S5.8), for reasons that remain unclear.

Cooperativity: The dramatic increase in swelling for $\mathrm{p}(\mathrm{AAm}-\mathrm{co}-\mathrm{AA}-\mathrm{co}-2)$ hydrogels above $\mathrm{pH}$ 5.5 may be a result of chemo-mechanical (in which chemistry controls the mechanics of a material) and mechano-chemical feedback (in which mechanics influences chemistry). As the internal $\mathrm{pH}$ increases above the $\mathrm{p} K_{\mathrm{a}}$ of acrylic acid, $\mathrm{p}(\mathrm{AAm}-\mathrm{co}-\mathrm{AA}-\mathrm{co}-2)$ hydrogels swell, inducing ring opening and converting SP (which is favored at higher $\mathrm{pH}^{47}$ ) to $\mathbf{M C H}^{+}$, increasing osmotic pressure and hydrophilicity and causing further swelling (positive feedback). As $\mathrm{pH}$ rises further, the SP/MC/MCH+-isomerization equilibrium shifts away from $\mathbf{M C H}^{+}$, leading to lower osmotic pressure and some shrinkage. The slight decrease in swelling ratios at higher $\mathrm{pH}$ could also be a result of the overall increase in salt concentration in the phosphate buffer solutions, ${ }^{50}$ (Figs. S5.12, S5.32 \& S6.1). In contrast, for $\mathrm{p}(\mathrm{AAm}-\mathrm{Co}$-DMAEMA-co-2) hydrogels that swell at low $\mathrm{pH}$, when spiropyran 2 mostly exists as $\mathbf{M C H}^{+}$, this effect is neither expected nor observed.

Aggregate formation: It is notable that the spiropyran-crosslinked hydrogels (Fig $\mathbf{4 b}$ ) do not show any formation of the $\lambda_{\max } \sim 470 \mathrm{~nm}$ absorption band observed in $\mathrm{p}(\mathrm{AAm}-\mathrm{co}-\mathrm{AA}-\mathrm{co}-1)$ and $\mathrm{p}(\mathrm{AAm}-\mathrm{Co}$-DMAEMA-co-1). Initial evidence of the possible nature of the species responsible for this absorption band comes from experiments in which $\mathrm{p}(\mathrm{AAm}-\mathrm{co}-\mathrm{DMAEMA-co-1)}$ hydrogel volume changes across the $\mathrm{pH} 3-8$ range are suppressed by increasing the salt concentration (200 mM $\mathrm{NaCl}$, Fig. S5.10 \& S5.31). Under such conditions, the band at $\lambda \sim 470 \mathrm{~nm}$ is present across the $\mathrm{pH}$ range. The 'salting out' effect of increased salt concentration may promote aggregation of the spiropyran appendages in ways that are sterically prevented when spiropyran acts as a crosslinker, thus potentially influencing the swelling behavior of the gels (Fig. 2). Merocyanines are well-known to form $\mathrm{H}$ - and J-aggregates. While the $\mathbf{M C H}^{+}$isomer has a generally lower tendency for aggregation, $\mathbf{M C}$ isomers form blue-shifted $\mathrm{H}$-aggregates with absorption maxima in the range of $\lambda$ $=440-480 \mathrm{~nm} .{ }^{55-57}$ (In p(AAm-co-1) gels, where no additional charges on the backbone are present, that could act as highly concentrated salts and contribute to the 'salting out' effect, no band at $\lambda_{\max } \sim 470 \mathrm{~nm}$ is observed.) Aggregated 1-MC could effectively sequester a significant amount of MC isomers, increasing the hydrophilicity of the polymer backbone and reducing the amount of charge from $\mathbf{M C H}^{+}$that could influence gel swelling. In contrast, spiropyran 2 could be too sterically encumbered to form aggregates and thus have more dye available to enhance swelling. There is also the possibility that such aggregates act as further crosslinking points in the hydrogel, which would reduce swelling, yet the magnitude of such an effect is difficult to estimate. Finally, we note that the presence of spiropyran aggregates may have significant effects on non-linear optical phenomena such as beam-trapping, which are under investigation in our laboratory. ${ }^{44,45}$ 


\section{Conclusion}

In conclusion, we report that spiropyran crosslinkers in polyelectrolytes can lead to unexpectedly large amounts of swelling compared to equivalent hydrogels with pendant spiropyran moieties. Nanoindentation experiments demonstrate that spiropyran-containing hydrogels have a lower elastic modulus than non-functionalized controls and that employing spiropyran 2 (crosslinker) instead of 1 (appended) increases the relative crosslinking density while also increasing hydrogel swelling. Solution $\mathrm{pH}$, buffering through co-monomers, ionic strength, and polymer strain through hydrogel swelling all influence the isomer distribution of spiropyran, some of which are charged and thus influence counterion pressure and swelling of the gel. However, our results suggest that counterion pressures alone are not sufficient to explain the observed swelling effects. Charge complexation, cooperative effects between mechanical strain and chemical changes in the hydrogel, and aggregate formation may all be invoked to explain features of the observed 'noninnocence' of spiropyran crosslinkers. However, more research will be required to better understand the specific mechanisms by which spiropyran crosslinkers affect hydrogel dynamics. Ultimately, better understanding the origin and role of such phenomena contributes novel insights into the function of an already well-established photoswitch within materials science and provides a new perspective to understand and design dynamic soft materials.

\section{Acknowledgements}

We would like to acknowledge financial support by the Department of Energy (DOE), Office of Science, Basic Energy Sciences (BES) under Award Number DE-SC0005247. M.M.L. was supported by the Netherlands Organization for Scientific Research (NWO, Rubicon Fellowship 019.182EN.027), and T.B.H.S was supported by the Swiss National Science Foundation (Postdoc.Mobility fellowship P400P2-180743). We would like to thank Drs. Elijah Shirman, Alison Grinthal, Jiaxi Ciu, Keti Piradeshvelli, Wendong Wong, and Oktay Uzun for fruitful discussions.

\section{Author contributions}

J.A. and A.S. conceived and initiated the project. A.S. synthesized the spiropyran compounds and hydrogel samples, measured their swelling behavior, mechanical properties, and conducted all UVvisible spectroscopic experiments. M.M.L. conducted follow-up studies to verify observed trends. A.S., M.M.L., T.B.H.S., A.M., M.A., A.V.S., and S.L. performed data analysis and interpretation of results. M.M.L. and J.A. supervised the project. A.S., A.M., T.B.H.S. and M.M.L. wrote the manuscript with contribution from all co-authors. All authors have given approval to the final version of the manuscript. 


\section{Competing Interests}

The authors declare no competing financial interest.

\section{Supplementary Information}

The following supplementary materials are available online:

\section{References}

(1) Philippova, O. Responsive Polymer Gels. Polym. Sci. - Ser. C 2000, 42, 208-228.

(2) Banerjee, H.; Suhail, M.; Ren, H. Hydrogel Actuators and Sensors for Biomedical Soft Robots: Brief Overview with Impending Challenges. Biomimetics 2018, p 15.

(3) Ionov, L. Biomimetic Hydrogel-Based Actuating Systems. Adv. Funct. Mater. 2013, 23 (36), 45554570.

(4) Ionov, L. Polymeric Actuators. Langmuir 2015, 31 (18), 5015-5024.

(5) Liu, X.; Liu, J.; Lin, S.; Zhao, X. Hydrogel Machines. Mater. Today 2020, 36, 102-124.

(6) Zhang, X.; Chen, L.; Lim, K. W. H. W. H.; Gonuguntla, S.; Lim, K. W. H. W. H.; Pranantyo, D.; Yong, W. P.; Yam, W. J. T.; Low, Z.; Teo, W. J.; et al. The Pathway to Intelligence: Using StimuliResponsive Materials as Building Blocks for Constructing Smart and Functional Systems. Adv Mater 2019, 31 (11), e1804540.

(7) Dong, L.; Jiang, H. Autonomous Microfluidics with Stimuli-Responsive Hydrogels. Soft Matter 2007, $3(10), 1223-1230$.

(8) Beebe, D. J.; Moore, J. S.; Bauer, J. M.; Yu, Q.; Liu, R. H.; Devadoss, C.; Jo, B.-H. Functional Hydrogel Structures for Autonomous Flow Control inside Microfluidic Channels. Nature 2000, 404 (6778), 588-590.

(9) Goy, C. B.; Chaile, R. E.; Madrid, R. E. Microfluidics and Hydrogel: A Powerful Combination. React. Funct. Polym. 2019, 145, 104314.

(10) Qian, X.; Zhao, Y.; Alsaid, Y.; Wang, X.; Hua, M.; Galy, T.; Gopalakrishna, H.; Yang, Y.; Cui, J.; Liu, N.; et al. Artificial Phototropism for Omnidirectional Tracking and Harvesting of Light. Nat. Nanotechnol. 2019, 14 (11), 1048-1055.

(11) Kirillova, A.; Maxson, R.; Stoychev, G.; Gomillion, C. T.; Ionov, L. 4D Biofabrication Using ShapeMorphing Hydrogels. Adv. Mater. 2017, 29 (46), 1703443.

(12) Sydney Gladman, A.; Matsumoto, E. A.; Nuzzo, R. G.; Mahadevan, L.; Lewis, J. A. Biomimetic 4D Printing. Nat. Mater. 2016, 15, 413.

(13) Biria, S.; Morim, D. R.; An Tsao, F.; Saravanamuttu, K.; Hosein, I. D. Coupling Nonlinear Optical Waves to Photoreactive and Phase-Separating Soft Matter: Current Status and Perspectives. Chaos An Interdiscip. J. Nonlinear Sci. 2017, 27 (10), 104611.

(14) Yuk, H.; Lu, B.; Zhao, X. Hydrogel Bioelectronics. Chem. Soc. Rev. 2019, 48 (6), 1642-1667.

(15) Yang, C.; Suo, Z. Hydrogel lonotronics. Nat. Rev. Mater. 2018, 3 (6), 125-142.

(16) Li, J.; Mooney, D. J. Designing Hydrogels for Controlled Drug Delivery. Nat. Rev. Mater. 2016, 1 (12), 16071.

(17) Sutton, A.; Shirman, T.; Timonen, J. V. I.; England, G. T.; Kim, P.; Kolle, M.; Ferrante, T.; Zarzar, L. D.; Strong, E.; Aizenberg, J. Photothermally Triggered Actuation of Hybrid Materials as a New Platform for in Vitro Cell Manipulation. Nat. Commun. 2017, 8, 14700.

(18) Vining, K. H.; Mooney, D. J. Mechanical Forces Direct Stem Cell Behaviour in Development and Regeneration. Nat. Rev. Mol. Cell Biol. 2017, 18 (12), 728-742.

(19) Ziółkowski, B.; Florea, L.; Theobald, J.; Benito-Lopez, F.; Diamond, D. Self-Protonating SpiropyranCo-NIPAM-Co-Acrylic Acid Hydrogel Photoactuators. Soft Matter 2013, 9 (36), 8754-8760.

(20) Li, C.; Iscen, A.; Palmer, L. C.; Schatz, G. C.; Stupp, S. I. Light-Driven Expansion of Spiropyran Hydrogels. J. Am. Chem. Soc. 2020, 142 (18), 8447-8453.

(21) Aizenberg, M.; Okeyoshi, K.; Aizenberg, J. Inverting the Swelling Trends in Modular Self-Oscillating Gels Crosslinked by Redox-Active Metal Bipyridine Complexes. Adv. Funct. Mater. 2017, 1704205.

(22) Zhang, Y.; Zhou, N.; Akella, S.; Kuang, Y.; Kim, D.; Schwartz, A.; Bezpalko, M.; Foxman, B. M.; Fraden, S.; Epstein, I. R.; et al. Active Cross-Linkers That Lead to Active Gels. Angew. Chem. Int. Ed. 2013, 52 (44), 11494-11498. 
(23) Kortekaas, L.; Browne, W. R. The Evolution of Spiropyran: Fundamentals and Progress of an Extraordinarily Versatile Photochrome. Chem. Soc. Rev. 2019, 48 (12), 3406-3424.

(24) Klajn, R. Spiropyran-Based Dynamic Materials. Chem. Soc. Rev. 2014, 43 (1), 148-184.

(25) Minkin, V. I. Photo-, Thermo-, Solvato-, and Electrochromic Spiroheterocyclic Compounds. Chem. Rev. 2004, 104 (5), 2751-2776.

(26) Lukyanov, B. S.; Lukyanova, M. B. Spiropyrans: Synthesis, Properties, and Application. (Review). Chem. Heterocycl. Compd. 2005, 41 (3), 281-311.

(27) Berkovic, G.; Krongauz, V.; Weiss, V. Spiropyrans and Spirooxazines for Memories and Switches. Chem. Rev. 2000, 100 (5), 1741-1754.

(28) Li, J.; Nagamani, C.; Moore, J. S. Polymer Mechanochemistry: From Destructive to Productive. Acc. Chem. Res. 2015, 48 (8), 2181-2190.

(29) Akbulatov, S.; Boulatov, R. Experimental Polymer Mechanochemistry and Its Interpretational Frameworks. ChemPhysChem 2017, 18 (11), 1422-1450.

(30) Shi, Z.; Peng, P.; Strohecker, D.; Liao, Y. Long-Lived Photoacid Based upon a Photochromic Reaction. J. Am. Chem. Soc. 2011, 133 (37), 14699-14703.

(31) Liao, Y. Design and Applications of Metastable-State Photoacids. Acc. Chem. Res. 2017, 50 (8), 1956-1964.

(32) Kollarigowda, R. H.; Braun, P. V. Direct and Divergent Solid-Phase Synthesis of Azobenzene and Spiropyran Derivatives. J. Org. Chem. 2021, 86 (6), 4391-4397.

(33) Feeney, M. J.; Thomas, S. W. Tuning the Negative Photochromism of Water-Soluble Spiropyran Polymers. Macromolecules 2018, 51 (20), 8027-8037.

(34) Aldoshin, S. M. Spiropyrans: Structural Features and Photochemical Properties. Mol. Cryst. Liq. Cryst. Sci. Technol. Sect. A. Mol. Cryst. Liq. Cryst. 1994, 246 (1), 207-214.

(35) Reichardt, C. Solvatochromic Dyes as Solvent Polarity Indicators. Chem. Rev. 1994, 94 (8), 2319 2358.

(36) Day, J. H. Thermochromism. Chem. Rev. 1963, 63 (1), 65-80.

(37) Seeboth, A.; Lötzsch, D.; Ruhmann, R.; Muehling, O. Thermochromic Polymers-Function by Design. Chem. Rev. 2014, 114 (5), 3037-3068.

(38) Molecular Switches, 2nd ed.; Feringa, B. L., Browne, W. R., Eds.; Wiley-VCH: Weinheim, Germany, 2011.

(39) Natali, M.; Giordani, S. Molecular Switches as Photocontrollable "Smart" Receptors. Chem. Soc. Rev. 2012, 41 (10), 4010-4029.

(40) Stafforst, T.; Hilvert, D. Kinetic Characterization of Spiropyrans in Aqueous Media. Chem. Commun. 2009, 0 (3), 287-288.

(41) Hammarson, M.; Nilsson, J. R.; Li, S.; Beke-Somfai, T.; Andréasson, J. Characterization of the Thermal and Photoinduced Reactions of Photochromic Spiropyrans in Aqueous Solution. J. Phys. Chem. B 2013, 117 (43), 13561-13571.

(42) Davis, D. A.; Hamilton, A.; Yang, J.; Cremar, L. D.; Van Gough, D.; Potisek, S. L.; Ong, M. T.; Braun, P. V; Martínez, T. J.; White, S. R.; et al. Force-Induced Activation of Covalent Bonds in Mechanoresponsive Polymeric Materials. Nature 2009, 459 (7243), 68-72.

(43) Magrini, T.; Kiebala, D.; Grimm, D.; Nelson, A.; Schrettl, S.; Bouville, F.; Weder, C.; Studart, A. R. Tough Bioinspired Composites That Self-Report Damage. ACS Appl. Mater. Interfaces 2021, 13 (23), 27481-27490.

(44) Morim, D. R.; Meeks, A.; Shastri, A.; Tran, A.; Shneidman, A. V; Yashin, V. V; Mahmood, F.; Balazs, A. C.; Aizenberg, J.; Saravanamuttu, K. Opto-Chemo-Mechanical Transduction in Photoresponsive Gels Elicits Switchable Self-Trapped Beams with Remote Interactions. Proc. Natl. Acad. Sci. 2020, 117 (8), 3953 LP - 3959.

(45) Meeks, A.; Mac, R.; Chathanat, S.; Aizenberg, J. Tunable Long-Range Interactions between SelfTrapped Beams Driven by the Thermal Response of Photoresponsive Hydrogels. Chem. Mater. 2020, 32 (24), 10594-10600.

(46) Doi, M.; Matsumoto, M.; Hirose, Y. Deformation of lonic Polymer Gels by Electric Fields. Macromolecules 1992, 25 (20), 5504-5511.

(47) Berton, C.; Busiello, D. M.; Zamuner, S.; Solari, E.; Scopelliti, R.; Fadaei-Tirani, F.; Severin, K.; Pezzato, C. Thermodynamics and Kinetics of Protonated Merocyanine Photoacids in Water. Chem. Sci. 2020, 11 (32), 8457-8468.

(48) Laurence, J. S.; Nelson, B. N.; Ye, Q.; Park, J.; Spencer, P. Characterization of Acid-Neutralizing Basic Monomers in Co-Solvent Systems by NMR. Int. J. Polym. Mater. Polym. Biomater. 2014, 63 
(7), 361-367.

(49) Lee, C. K.; Diesendruck, C. E.; Lu, E.; Pickett, A. N.; May, P. A.; Moore, J. S.; Braun, P. V. Solvent Swelling Activation of a Mechanophore in a Polymer Network. Macromolecules 2014, 47 (8), 26902694.

(50) Longo, G. S.; Olvera de la Cruz, M.; Szleifer, I. Molecular Theory of Weak Polyelectrolyte Gels: The Role of PH and Salt Concentration. Macromolecules 2011, 44 (1), 147-158.

(51) Longo, G. S.; Olvera de la Cruz, M.; Szleifer, I. Controlling Swelling/Deswelling of Stimuli-Responsive Hydrogel Nanofilms in Electric Fields. Soft Matter 2016, 12 (40), 8359-8366.

(52) Li, H.; Erbaş, A.; Zwanikken, J.; Olvera de la Cruz, M. lonic Conductivity in Polyelectrolyte Hydrogels. Macromolecules 2016, 49 (23), 9239-9246.

(53) Erbas, A.; Olvera de la Cruz, M. Energy Conversion in Polyelectrolyte Hydrogels. ACS Macro Lett. 2015, 4 (8), 857-861.

(54) Nakajima, T.; Hoshino, K. I.; Guo, H.; Kurokawa, T.; Gong, J. P. Experimental Verification of the Balance between Elastic Pressure and Ionic Osmotic Pressure of Highly Swollen Charged Gels. Gels 2021, 7 (2), 39.

(55) Eckhardt, H.; Bose, A.; Krongauz, V. A. Formation of Molecular H- and J-Stacks by the SpiropyranMerocyanine Transformation in a Polymer Matrix. Polymer 1987, 28 (11), 1959-1964.

(56) Wagner, K.; Byrne, R.; Zanoni, M.; Gambhir, S.; Dennany, L.; Breukers, R.; Higgins, M.; Wagner, P.; Diamond, D.; Wallace, G. G.; et al. A Multiswitchable Poly(Terthiophene) Bearing a Spiropyran Functionality: Understanding Photo- and Electrochemical Control. J. Am. Chem. Soc. 2011, 133 (14), 5453-5462.

(57) McCoy, C. P.; Donnelly, L.; Jones, D. S.; Gorman, S. P. Synthesis and Characterisation of Polymerisable Photochromic Spiropyrans: Towards Photomechanical Biomaterials. Tetrahedron Lett. 2007, 48 (4), 657-661. 